\title{
COVID-19 and the Brain
}

\section{Maria Elena López $^{1 *}$ and Sofia Isea ${ }^{2}$}

${ }^{1}$ Pediatrics Department of Luanda Medical Center, Consultant, Venezuela

${ }^{2}$ Surgeon Physician Attached to the Medical Assistance Residency of Centro Medico

Docente La Trinidad, Venezuela

*Corresponding Author: Maria Elena López, Pediatrics Department of Luanda

Medical Center.
Received: July 19, 2020

Published: August 25, 2020

(C) All rights are reserved by Maria Elena

López and Sofia Isea.

\begin{abstract}
During the present pandemic of COVID-19 disease, interest has shifted from the respiratory system affectation to involvement of other organs and systems. Recently pre-eminence of neurological symptoms and signs has emerged. We have done a comprehensive review of the literature published as open access in various databases with the purpose of increasing our understanding of the disease and aid in the identification of patients with these neurological conditions. We have reviewed articles reporting neurologic manifestations in young individuals and children. Although there is nor enough data to establish prevalence of the conditions affecting pediatric population, as the number of worldwide cases increases we expect to find an increase in these reports. it is considered of utmost importance to urge clinicians to document symptoms and neurological signs in the context of the disease in order to contribute to a better understanding of Neurocovid and to generate a body of knowledge that influences the optimization of the diagnosis and approach of this entity, taking as a premise that the early installation of individualized and reasoned treatments will translate in a better survival rate of those affected and lower the occurrence of eventual ongoing deficits.
\end{abstract}

Keywords: COVID-19; Severe Acute Respiratory Syndrome (SAPS); Middle East Respiratory Syndrome (MERS)

\section{Introduction}

Since December 2019, concern has been raised about the emergence of cases related to infection by a capsular RNA virus of a helicoidal chain belonging to Class IV [1]. This family of viruses is known for its relationship with other agents that produce high-severity respiratory diseases such as SARS (Severe acute respiratory syndrome) and MERS (Middle East respiratory syndrome). Due to these close similarities it has been called SARS-CoV-2.

During the first quarter of 2020, the WHO recognizes the categorization of a pandemic due to the rapidly spreading number of cases identified worldwide. The high similarity of the viral genome with that of the bat coronavirus, has established that it is a pathogen originating from these animal species, with the subsequent presence of a non-human intermediate host [2].

It is known that the specular structural proteins of the viral capsid binds to the ACE- 2 receptors of the cells of lung tissue, and then, once released inside the cell, takes advantage of the intracellular mechanisms of protein synthesis for it's replication.

Although more attention was paid to the respiratory complications of the disease at the beginning of the pandemic, as the global number of cases has raised, an increasing number of patients affected by the new Coronavirus have been observed to present involvement of the nervous system in more of $40 \%-57 \%$ [3] of the cases. Manifestations of varying severity are described, ranging from headache, anosmia and dysgeusia [4], alterations in the state 
of consciousness, paralysis, paresthesias and convulsive symptoms [5], which may occur even after respiratory symptoms and in other cases in the absence of respiratory symptoms [6].

\section{Mechanisms of pathogenesis and models of approach}

Severe neurological involvement in relation to SARS-CoV-2 has been described with three categories based on the spectrum of clinical presentation consisting of Cerebral thrombosis. with hemorrhagic infarcts in 5.7\% of cases, demyelinating lesions [7] and encephalopathy $[8,9]$, which is why the term NEURO-COVID has been coined for these entities.

Among the proposed pathophysiological mechanisms of neurological involvement by COVID 19, it is known that the virus induces significant hypercytokinemia, with an increase in factors such as IL-6, IL-1 Beta, and Tumor Necrosis Factor TNF alpha [10]. This inflammatory process ends up generating vascular injury with disruption of the blood-brain barrier. Additionally, it has been described that in the presence of COVID-19 infection, helper T-cell imbalance is observed with an exaggerated increase in Th1 [11] responses, as well as direct invasion of the brain tissue [12].

An etiopathogenic model of neurological involvement by this agent has been proposed, which consists of three steps, these being Neuroinvasion, CNS clearance, and Immune Response. During the initial stage, SARS-CoV-2 reaches the brain through the bloodstream or through the cribriform plate of the ethmoid to follow the path of the olfactory nerve by axonal transport and reach the frontal cerebral cortex. Other authors have raised the possibility of SARS-CoV-2 entry through olfactory/cervical lymphatic vessels that can facilitate direct brain invasion [13]. During this first stage or phase, the viral load in the spinal brain fluid begins to rise progressively.

Through the interaction of the viral S1 specular protein and the host ACE-2 receptors, the entry of the virus into the neuronal interior is facilitated, causing direct damage to the nerve endings and generating the early symptoms of anosmia and dysgeusia of early disease. During the CNS clearance phase, the viral load decreases, however, in some patients the involvement of the structures of the brain stem occurs, and this process may be an aggravating event of severe respiratory symptoms due to nuclear invasion of the solitary tract and determining torpid evolution.
In the third phase the neocortex and the brain stem structures are colonized by the virus, with the consequent alteration of the state of consciousness and the eventual presence of seizures, coinciding with a level of greater severity of both direct and immune mediated by the increase of antibodies against glia cells as a para or post infectious phenomenon [14], which in the presence of hypoxia as an enhancer of endothelial damage, and the presence of vascular thrombosis leads to a response of multifactorial injury, sometimes catastrophic of the nervous tissue [15]. The classification of the level of damage is then proposed with the categories of NeuroCOVID I, NeuroCOVID II and NeuroCOVID III.

NeuroCOVID I being understood as the degree of damage limited to the epithelium of the nose, rhino and oropharynx, the main manifestation being loss of smell and taste. NeuroCOVID II corresponds to the activation of the exaggerated inflammatory response at the pulmonary level that we know as the storm of cytokines that pass into the bloodstream and spread throughout all tissues including the brain, triggering the presence of thrombi that can cause strokes of variable magnitude.

The category of NeuroCOVID III coincides with the exaggerated and sustained activation of inflammatory and defense mechanisms, maintaining and increasing the cytokine storm, disrupting the permeability of the brain capillaries with an increase of permeability of the blood-brain barrier, allowing a series of Inflammatory substances, viral particles and blood components to penetrate the brain causing even more severe damage, the clinical expression of which are seizures, changes in the sensory level, coma and encephalopathy [16].

Among the neurological entities described in the literature in relation to the pandemic, in addition to reports of the presence of anosmia, ageusia/dysgeusia, a report of cases with acute vestibular dysfunction has been found as an isolated manifestation [17]. Likewise, there is literature on cases of progressive tetraparesis and areflexia consistent with Guillain Barre Syndrome [18,19] with a torpid and rapidly progressive course, in the context of previous infection with mild symptoms or asymptomatic respiratory patients with SARS-CoV-2.

Likewise, cases have been described of patients with acute meningoencephalitis, clinically attributable to the virus or with confirmed presence of SARS-CoV-2 by genomic sequencing in ce- 
rebrospinal fluid $[20,21]$. Other authors point out that in severe cases, up to $40 \%$ of patients may present headache, changes in the level of consciousness and other neurological symptoms, finding in autopsy studies of these subjects show presence of cerebral edema [22], neuronal degeneration and meningeal vasodilation [23] without significant cerebrospinal fluid changes, which supports the occurrence of Toxic Infectious Encephalopathy.

SARS-CoV-2 infection has been widely documented not only as a cause of cytokine-mediated syndromes that may contribute to the installation of cerebral vascular disease [24], but additionally, a significant increase has been found in patients with SARS-CoV-2 of dimer $\mathrm{D}$ and other substances that support the thrombotic tendency in this pathology and are risk predictors for the presentation of acute events such as cerebral infarction [25] and at least one case of cerebral venous thrombosis [26]. On the other hand, knowing the protective role of the angiotensin-converting enzyme in a variety of organs and systems, it is understood that by binding the virus with ACE 2 receptors, abnormally high blood pressures can be caused, which together with the impact of the virus in the capillary endothelium and thrombocytopenia, can produce cerebral hemorrhagic injury [27].

A case of acute necrotizing hemorrhagic encephalopathy has been described as a catastrophic complication of SARS-CoV-2 infection, with neuroimaging findings consistent with involvement of both gray and white matter, with important changes in the basal ganglia and other structures of high metabolic rate, generally symmetrical and accompanied by equally bilateral hemorrhages with perilesional enhancement after contrast injection in the presence of undamaged regional vasculature elements, which although it is a pathology of extreme severity with a higher frequency of presentation in the pediatric population also occurs in adults contributing to further darken the prognosis of patients with neurological involvement during the pandemic.

There are reports in the consulted literature of patients whose only manifestation has been the presence of convulsive syndromes, epileptic status in patients with a history of epilepsy secondary to other conditions [28], describing a semi-rhythmic background activity, in the delta range with slow outbreaks in the left frontal-temporal center. Another case has been reported with electroencephalographic alterations consisting of slow global background activity with temporal focal activity due to outbreaks of hypervolted acute waves that are characteristic of an encephalopathic pattern with specific epileptogenic activity [29]. However, in a retrospective multicenter study, no evidence was found to suggest that the seizures seen in patients with COVID-19 disease are directly related to the SARS-CoV-2 virus, without underestimating the role of triggers such as structural lesions. Previous, equally preexisting cortical electrical dysfunctions and hypoxic conditions, hydroelectrolytic disturbances and high metabolic stress in critically ill patients [30].

\section{Special considerations in pediatric population}

Studies prior to the current pandemic report the occurrence of central nervous system infection by coronavirus in children. The authors draw attention to the neurotropism of these pathogens and have identified expression profiles of cytokines involved in the primary immune response as an inducer of brain damage in pediatric patients, in addition to pointing out that children, like adults, may experience neurological complications that will depend on environmental variables and factors inherent to the host, such as genetic alterations or immuno-mediated processes [31].

Also previously, other coronaviruses have been linked to some demyelinating entities of the central nervous system. Finding reports of acute encephalitis, flaccid paralysis and seizures; as well as at least one case report of a healthy 15-year-old boy with a normal neuroevolutionary history with a picture of Acute Disseminated Encephalomyelitis, in which it is possible to identify human coronavirus by polymerase chain reaction with a significant increase in the titers of antibodies in cerebrospinal fluid and in nasopharyngeal secretions [32].

Until now it has been weighted that the pediatric population has protective factors like a lower expression of ACE-2 receptors [33], the presence of cross immunity from previous infections by other viruses, the lower incidence of comorbidities or chronic underlying diseases and some immunological peculiarities that are thought to decrease the inflammatory tendency in children and young individuals.

A study in Wuhan evaluating 2143 pediatric cases, found that $5.9 \%$ of affected children presented severe disease with $0.6 \%$ evolving with multi-organ failure or Adult respiratory distress syndrome. Of these children with severe evolution, it was observed that $10.6 \%$ corresponded to the age range of children in the infant 
stage, and at the same time, those children with pre-existing conditions such as kidney disease and leukosis, as well as chronic respiratory diseases, are identified as a category of increased risk [34].

Another retrospective case review study in children ages 0 - 16 confirmed for COVID-19. A total of 36 cases were identified in three hospitals in Zhejiang city, of which $53 \%$ of the sample presented moderate pneumonia and the remaining $47 \%$ were categorized as mild cases (asymptomatic or with upper respiratory symptoms). In the group with pneumonia, they describe an increase in CKMB, an increase in procalcitonin and Dimer D and leukopenia with lymphopenia. These children were hospitalized and the authors do not report fatalities or neurological symptoms [35].

Likewise, during the surveillance of cases in the United States, the first pediatric case was reported on March 02 and by the beginning of April 2020, a total figure of 2,572 cases was reached in children under 18 years of age, corresponding to $1.7 \%$ of the total number of cases identified. Of these 2,572 cases, a distribution was observed in which it predominates with $32 \%$ of cases in patients between 15 and 17 years old, 27\% in the age range between 10 and 14 years, $15 \%$. In the range of 5 and 9 years, $11 \%$ in children from 1 to 4 years and $15 \%$ of children under one year of age, who are the most frequently hospitalized, together with children with underlying conditions. At the time of the aforementioned report (April 2020), three deaths had been registered and were being studied to identify a direct relationship with Sars-CoV-2 [35].

June 2020, it is established that in the group of North American children from 0 to 4 years of age, $1.9 \%$ of the fatalities due to the SARS-CoV-2 virus occur.

Although the data derived from the experience in China, as well as recent $\mathrm{CDC}$ reports regarding the COVID-19 pandemic, continue to indicate that the disease occurs with fewer manifestations and severity in the population under 18 years of age. As world casuistry rises we are faced with an increase in pediatric cases. However, until recently, insufficient epidemiological data are available to establish the true prevalence of neurological disease in children as a consequence of COVID-19, including anosmia, dysgeusia [36], cerebrovascular events, disorders of consciousness, cranial nerve dysfunction, encephalopathy. acute hemorrhagic necrotizing and Guillain Barré syndrome.
In recent weeks, new research works appear, among which a study in the city of London stands out, which analyzes the data from electronic files of 27 children who attended between March and May 2020 and were confirmed with Multisystemic Inflammatory Syndrome associated with COVID-19. Of this group of patients, 4 children presented de novo neurological symptoms. The 4 patients in this study presented early in the course of the multisystemic Inflammatory Syndrome, decrease in proximal muscle strength and hyporeflexia. Additionally, signs of encephalopathy, headache and brain stem dysfunction (difficulties in the articulation of language and dysphagia), ataxia and other signs of cerebellar involvement were identified.

Magnetic resonance findings are characterized by changes in the splenium of the corpus callosum were observed in the 4 patients, similar to those seen in other pathologies, which have been related to possible intramyelin focal edema and have been reported in patients with ischemia, after reversible encephalopathies and in patients with severe hydroelectrolytic disorders, in addition to some patients with Kawasaki syndrome [37].

\section{Conclusion}

In order to correct the paucity of data and increase the understanding of the pathology in both pediatric and adult populations, prospective cohort follow-up multi-centric studies are currently being implemented [38,39] and it is considered of utmost importance to urge clinicians to document symptoms and neurological signs in the context of the disease in order to contribute to a better understanding of Neurocovid and to generate a body of knowledge that influences the optimization of the diagnosis and approach of this entity, taking as a premise that the early installation of individualized and reasoned treatments will translate in a better survival rate of those affected and lower the occurrence of motor, sensitive, cognitive sequential deficits that, although they observed so far in adults and seem not to have been identified in children, begin to occupy the attention of the scientific community.

\section{Bibliography}

1. Baltimore D. "Expression of animal virus genomes". Bacteriology Review 35 (1971): 235-24.

2. Chan J., et al. "Genomic characterization of the 2019 novel human -pathogenic coronavirus isolated from a patient with 
atypical pneumonia after visiting Wuhan". Emerging Microbes and Infections 28.9 (2020): 221-236.

3. Romero-Sánchez C., et al. "Neurologic Manifestations in hospitalized patients with COVID-19: The ALBACOVID registry". Neurology (2020).

4. Vavougios G. "Potentially irreversible olfactory and gustatory impairments in Covid-19: Indolent versus Fulminant SARSCoV-2 neuroinfection". Brain, Behavior, and Immunity (2020).

5. Mao L., et al. "Neurological Manifestations of Hospitalized Patients with COVID-19 in Wuhan, China: a retrospective case series study". Lancet Neurology (2020).

6. Bertran-Recasens B., et al. "Lack of dyspnea in Covid-19 patients another neurological conundrum?" European Journal of Neurology (2020).

7. Zanin L., et al. "SARS-COV-2 can induce brain and spine demyelinating lesions". Acta Neurochirurgica 4 (2020): 1-4.

8. Moriguchi T., et al. "A first case of Meningitis/Encephalitis associated with SARS-Coronavirus-2". International Journal of Infectious Diseases 94 (2020): 54-58.

9. Huang H., et al. "SARS-CoV-2 Detected in Cerebrospinal Fluid by PCR in a Case of COVID-19 Encephalitis". Brain, Behavior, and Immunity 87 (2020): 149.

10. Quin C., et al. "Dysregulation of immune response in patients with Covid-19 in Wuhan, China". China (February 17,2020) (2020).

11. Huang C., et al. "Clinical features of patients infected with 2019 novel coronavirus in Wuhan, China". The Lancet 395 (2020): 497-506.

12. Duong L., et al. "Meningoencephalitis without respiratory failure in a young female patient with Covid-19 infection in downtown Los Angeles, early April 2020". Brain, Behavior, and Immunity 87 (2020): 33.

13. Varga Z., et al. "Endothelial cell infection and endothelitis in COVID 19”. Lancet 395.10234 (2020): 1417-1418.

14. Kanberg N., et al. "Neurochemical evidence of astrocytic and neuronal in jury commonly found in COVID-19". Neurology (2020).
15. Panciani P., et al. "SARS-CoV-2: "Three-steps" infection model and CSF diagnostic implication". Brain, Behavior, and Immunity (2020).

16. Fotuhi M., et al. "Neurobiology of COVID-19". Journal of Alzheimer's Disease (2020): 1-17.

17. Escalada-Pellitero S., et al. "Report of a patient with neurological symptoms as the sole manifestation of SARS-Cov-2 infection". Madrid. Spain (2020).

18. Zhao H., et al. "Guillain Barre Syndrome associated with SARSCoV-2 infection: Causality or coincidence?" Lancet Neurology 19.5 (2020): P383-384.

19. El Otmani H., et al. "Covid-19 and Guillain-Barré syndrome: More than a coincidence!". Revue Neurologique 176 (2020): 516-525.

20. Chaumont H., et al. "Acute Meningoencephalitis in a patient with COVID-19”. Revue Neurologique 176 (2020): 516-525

21. Xiang P., et al. "First case of 2019 novel coronavirus disease with Encephalitis". ChinaXiv (2020).

22. Xu Z., et al. "Pathological findings of COVID-19 associated with acute respiratory distress syndrome". Lancet Respiratory Medicine 8.4 (2020): 420-422.

23. Wu Y., et al. "Nervous system involvement after infection with COVID-19 and other Coronaviruses". Brain Behavior and Immunity (2020).

24. Mehta P., et al. "COVID-19: consider cytokine storm syndromes and immunosuppression". Lancet 395.10229 (2020): 10331034.

25. Reyes M., et al. "COVID-19 and D-dimer: Frequently asked questions". COVID-19 Resources @hematology.org. American Society of Hematology (2020).

26. Hemasian H and Ansari B. "First case of Covid-19 presented with cerebral venous thrombosis: A rare and dreaded case". Revue Neurologique 176 (2020) 516-525.

27. Baig A., et al. "Evidence of COVID-19 virus targeting the CNS: tissue distribution, host virus interactions, and proposed neurotropic mechanisms". ACS Chemical Neuroscience 11.7 (2020): 995-998. 
28. Vollono C., et al. "Focal Status epilepticus as a unique clinical feature of COVID/19: a case report". Our Journal of Epilepsy (2020).

29. Filatov A., et al. "Neurological Complications of Corona virus Disease (COVID-19): Encephalopathy". Cureus 12.3 (2020): e7352.

30. Lu l., et al. "New onset acute symptomatic seizure and risk factors in coronavirus disease 2019: a multi center retrospective study". Epilepsia 61.9 (2020): e49-e53.

31. Li Y., et al. "Coronavirus infections in the central nervous system and respiratory tract show distinct features in hospitalized children". Intervirology 59 (2016): 163-169.

32. Yeh EA., et al. "Detection of Coronavirus in the Central Nervous System of a Child With Acute Disseminated Encephalomyelitis". Pediatrics 113.1 (2004)e73-e76.

33. Bunyavanich S., et al. "Nasal gene expression of angiotensinconverting enzyme 2 in children and adults". JAMA 323.23 (2020): 2427-2449.

34. Dong Y., et al. "Epidemiological Characteristics of 2143 pediatric patients with 2019 coronavirus disease in China". Pediatrics (2020): e20200702.

35. Qiu H., et al. "Clinical and epidemiological features of $36 \mathrm{chil}-$ dren with coronavirus disease 2019 (COVID-19) in Zhejiang, China: an observational cohort study". The Lancet Infectious diseases 20 (2020): 689-696.

36. Vaira L., et al. "Anosmia and ageusia: common findings in COVID-19 patients". Laryngoscope (2020).

37. Abdel-Mannou O., et al. "Neurological and Radiographic Findings Associated with COVID-19 in Children". JAMA Neurology (2020).

38. Frontera J., et al. "Global Consortium Study of Neurological Dysfunction in COVID-19 (GCS-NeuroCOVID): Study Design and Rationale". Neurocritical Care (2020): 1-10.

39. Neurocritical Care Society. "Covid-19 Manifestations in Pediatric Patients" (2020).

\section{Assets from publication with us}

- Prompt Acknowledgement after receiving the article

- Thorough Double blinded peer review

- Rapid Publication

- Issue of Publication Certificate

- High visibility of your Published work

Website: www.actascientific.com/

Submit Article: www.actascientific.com/submission.php

Email us: editor@actascientific.com

Contact us: +919182824667 